\title{
Construção e Implantação de uma Metodologia Ágil no Processo de Desenvolvimento de Software de uma Organização Publica: Uma Pesquisa-Ação
}

\author{
Jéssica S. Garcia ${ }^{1}$, Joyce Aline Marins ${ }^{1}$, Vanice Cunha ${ }^{2}$, Aline Wollinger ${ }^{3}$, \\ Giulla Araújo ${ }^{3}$ \\ ${ }^{1}$ Faculdade de Engenharia - Universidade Federal do Mato Grosso (UFMT) \\ Cuiabá - MT - Brasil. \\ ${ }^{2}$ Instituto de Computação - Universidade Federal do Mato Grosso (UFMT) \\ Cuiabá - MT - Brasil. \\ ${ }^{3}$ Tribunal de Contas do Estado de Mato Grosso (TCE-MT) \\ Cuiabá - MT - Brasil. \\ jessicagarcia132012@hotmail.com, joyce.marins@ufmt.br \\ vanice@ic.ufmt.br,\{aline,giulla\}@tce.mt.gov.br
}

\begin{abstract}
The Court of Auditors of the State of Mato Grosso has adopted a methodology based on the SCRUM. It is noticeable that the use of the new methodology has promoted an increase in the quality of the systems, reduced implementation time and increased communication and transparency. This article reports the construction and implementation of this methodology, which was built through action research and evaluated from the perspective of the development team. The challenges reported and lessons learned are of great relevance for organizations that aim to change the traditional development paradigm to agile in their contexts.
\end{abstract}

Resumo. O Tribunal de Contas do Estado do Mato Grosso tem adotado uma metodologia baseada no SCRUM. É perceptível que o uso da nova metodologia promoveu o aumento da qualidade dos sistemas, diminuiu o tempo de implementação e aumentou a comunicação e a transparência. Este artigo relata o construção e implantação desta metodologia que foi construída por meio de uma pesquisa-ação e avaliada sob a perspectiva da equipe de desenvolvimento. Os desafios relatados e as lições aprendidas são de grande relevância para organizações que visam mudar o paradigma de desenvolvimento tradicional para ágil em seus contextos.

\section{Introdução}

Ambientes de projetos possuem incertezas e dificuldades que podem ser resolvidas por meio de métodos tradicionais, ágeis ou híbridos [Oliveira and Pedron 2021]. Diversas evidências na literatura mostram a melhoria do desempenho de projetos no âmbito da equipe, satisfação do cliente, eficiência e eficácia do processo, custo, prazo e funcionalidades do projeto com o uso de metodologias ágeis. Segundo [Nicholls et al. 2015], métodos ágeis foram desenvolvidos para aumentar a eficiência, produtividade e capacidade de resposta para o cliente. Este paradigma possui foco na simplicidade, nas pessoas 
e no aumento da produtividade. Um dos métodos ágeis amplamente utilizado por equipes de desenvolvimento de software é o Scrum, que é conhecido por sua simplicidade, por exigir pouca documentação de requisitos, pela facilidade de aprendizagem e pela flexibilidade quanto a regras [Schwaber 2004]. Esse método possui como estrutura um conjunto de práticas que mantém o seu passo a passo visível a todos, para que toda a equipe tenha ciência do estágio em que está o projeto e quais mudanças deverão ser executadas a fim de alcançar as metas desejadas [Oliveira and Pedron 2021].

Segundo [Cooper 2008], embora metodologias ágeis apresentam inúmeras vantagens, há dificuldades e desafios associados à sua implementação principalmente em equipes que estão acostumadas com metodologias tradicionais. $\mathrm{Na}$ literatura são encontrados vários trabalhos que indicam dificuldades de adoção de métodos ágeis principalmente em ambientes do setor público. As principais limitações mencionadas são: estrutura hierárquica, formalidade, limitação de pessoal, necessitando que nesses ambientes ocorra uma mudança de cultura, relações e rotinas [Oliveira and Pedron 2021, Noordeloos et al. 2012, Lappi and Aaltonen 2017, Hidalgo 2019, de Souza Carvalho et al. 2011]. Portanto, a mudança do paradigma de desenvolvimento tradicional para o paradigma ágil precisa ser cuidadosamente planejada.

Este artigo relata a construção e implantação de uma metodologia ágil baseada no SCRUM que foi construída e aplicada no Tribunal de Contas do Estado de Mato Grosso por meio de uma pesquisa-ação. A metodologia construída foi chamada de PDMS ágil (Processo de Desenvolvimento e Manutenção de Software) e possui as características, os artefatos e os papéis baseados no SCRUM. O TCE-MT antes trabalhava com uma metodologia tradicional baseada no RUP (Rational Unified Process). O Rational Unified Process (RUP) é uma estrutura popular de desenvolvimento de software iterativo e incremental, onde os processos estruturados são caracterizados por um foco em práticas rigorosamente definidas, extensa documentação, planejamento e gerenciamento detalhados [Shafiee et al. 2020]. A dificuldade em gerir a grande quantidade de documentação, o atraso nas entregas das funcionalidades e a necessidade de atender com maior rapidez as demandas do setor de Tecnologia da Informação exigiram a evolução da metodologia anteriormente utilizada para um paradigma ágil. Foi perceptível pela gestão e seus desenvolvedores que a adoção de uma metodologia ágil no TCE-MT aumentou a qualidade dos sistemas desenvolvidos e diminuíram o tempo de implementação. Como contribuição teórica, os resultados aumentaram o corpo de conhecimento sobre metodologias de desenvolvimento em engenharia de software. As implicações práticas serão viáveis por meio da adoção da metodologia por outras organizações.

\section{Método}

De acordo com [Baskerville 1999], a Pesquisa-Ação fundamenta-se na afirmação de que processos sociais complexos podem ser melhor estudados introduzindo-se mudanças e observando-se os efeitos destas no ambiente de aplicação. A flexibilidade da PesquisaAção possibilita criar e adaptar passos para orientar a condução desta abordagem. $\mathrm{O}$ conjunto destes passos denomina-se ciclo de vida. O ciclo de vida da pesquisa-ação adotado nesta investigação foi composto pelos seguintes passos:

- 1. Diagnóstico - Envolveu uma investigação na organização participante com o propósito de identificar características culturais e funções da equipe de desenvol- 
vimento e características da metodologia tradicional em vigor que era chamada de PDMS (Processo de Desenvolvimento e Manutenção de Software);

- 2. Planejamento - Foi elaborada a metodologia ágil com artefatos, papéis e atividades de acordo com as especificidades do SCRUM e foi chamada de PDMS ágil.

- 3. Implementação da ação - Gradualmente o PDMS ágil que é baseado no Scrum foi implantado nos projetos de desenvolvimento e as ferramentas necessárias, por exemplo o Redmine, foram configuradas para suportar o acompanhamento dos projetos de forma ágil.

- 4. Avaliação - Os resultados da implantação foram analisados por meio de uma entrevista semi-estruturada com a gestão com o propósito de avaliar a intervenção e os benefícios alcançados. A avaliação da metodologia construída no TCE-MT teve os seguintes objetivos: i) Observar se a metodologia construída é completa e adequada para a equipe de desenvolvimento do TCE-MT; ii) Observar como a equipe se adapta ao paradigma agil; iii) Identificar desafios associados à transição de metodologias.

- 5. Documentação do aprendizado - As lições aprendidas foram documentadas e compartilhadas com a equipe para melhoria e continuidade do uso da metodologia ágil.

Os procedimentos de coleta de dados utilizados durante a Pesquisa-Ação foram entrevistas semi-estruturadas e documentação [Merriam and Tisdell 2009]. Os dados foram tratados por meio de análise temática.

\section{Resultados}

\subsection{Diagnóstico}

O TCE utilizava uma metodologia chamada de PDMS 11 (Processo de Desenvolvimento e Manutenção de Software). Esta metodologia era baseada no RUP e possuía vários artefatos a serem entregues em cada fase de desenvolvimento.

Estas fases consistiam em 1. Concepção 2 durante este processo, os analistas de sistemas da equipe do projeto deveriam identificar todos os requisitos do produto a partir de reuniões e entrevistas de levantamento junto aos usuários; 2 Elaboração 3 ; esta fase envolvia uma análise detalhada sobre as necessidade e problemas gerais do projeto e a definição de como o sistema deveria ser desenvolvido em termos tecnológicos, considerando os requisitos, limitações e restrições identificados durante a fase de Concepção; 3. Construção ${ }^{4}$ : A fase de Construção era dividida em iterações de acordo com a necessidade identificada. A cada iteração será gerada e testada uma nova release do sistema, contendo os casos de uso implementados até aquele momento; 4. Transição 5. consistia em realizar a homologação da versão final do sistema por seus usuários. A homologação do sistema deveria ser realizada considerando os itens descritos no RRH - Relatório de Resultados de Homologação. O sistema homologado junto a seus usuários era disponibilizado para implantação em ambiente de produção.

\footnotetext{
1 https://shortest.link/1obo

2 https://shortest.link/1jpv

3 https://shortest.link/1n5g

4 https://shortest.link/1n5j

5 https://shortest.link/1jpB
} 
Os artefatos a serem entregues em cada fase são mostrados na Tabela 1. Vários motivos tornaram clara a necessidade de o TCE mudar da metodologia tradicional, anteriormente utilizada para uma metodologia ágil, como falta de visibilidade dos projetos, dificuldade de gerenciamento do conhecimento, aumento de fluxo de trabalho e por consequência, dificuldade em gerir prazos para entrega das funcionalidades. A gestora responsável pela equipe de TI relatou que o TCE estava com dificuldades de obter informações relacionadas ao andamento dos projetos que estavam sendo realizados. Essas informações não eram armazenadas e não tinham onde e nem como buscá-las. Considerando estes problemas, a quantidade e fluxo de trabalho e a carência de gerir e mensurar as informações de interesse, foi demandada a implementação de uma metodologia ágil para que se conseguisse fazer todo o acompanhamento dos projetos de desenvolvimento. O objetivo dessa mudança era realmente organizar o cenário no qual o TCE se encontrava:excesso de projetos, um profissional envolvido em vários projetos, projetos complexos com prazos para serem entregues e que não se conseguia fazer o gerenciamento de forma efetiva.

Tabela 1. Artefatos RUP

\begin{tabular}{|l|l|}
\hline Fases & Artefatos \\
\hline Fase 1 & $\begin{array}{l}\text { Documento de Visão - estabelece as principais funcionalidades e fron- } \\
\text { teiras do sistema e registra informações que delimitam o projeto de } \\
\text { desenvolvimento como um todo. Funciona como um contrato entre } \\
\text { o TCE-MT e os demais interessados. Glossário - registra termos es- } \\
\text { pecíficos ao domínio do sistema e do projeto e funciona como um di- } \\
\text { cionário para a equipe de desenvolvimento. }\end{array}$ \\
\hline Fase 2 & $\begin{array}{l}\text { Matriz de Requisitos do Projeto através de um Diagrama de Casos de } \\
\text { Uso - registra todos os requisitos e as regras de negócios do sistema le- } \\
\text { vantados junto aos usuários gestores e finais. Modelo de casos de Uso e } \\
\text { Especificações de casos de Uso - O Modelo de Casos de Uso descreve } \\
\text { toda a visão funcional do sistema através de seus atores e casos de uso. } \\
\text { Documento de Arquitetura - descreve as principais decisões de projeto } \\
\text { tomadas pela equipe de desenvolvimento e os critérios considerados du- } \\
\text { rante a tomada destas decisões. }\end{array}$ \\
\hline Fase 3 3 & $\begin{array}{l}\text { Codificação do sistema - O código-fonte representa qualquer trecho } \\
\text { de código implementado ao longo do desenvolvimento do sistema. } \\
\text { Realização de testes unitários, de integração e compilação de versões } \\
\text { - A compilação do Sistema, ou build, representa o sistema executável } \\
\text { compilado. Release - representa o sistema executável ao final de uma } \\
\text { fase do projeto, representando o marco do ciclo de vida do projeto. Pa- } \\
\text { cote de Distribuição - representa o empacotamento físico de uma versão } \\
\text { do sistema, incluindo o sistema executável, kits de instalação, manuais } \\
\text { do sistema, documentação do projeto, bases de dados para carga, etc. }\end{array}$ \\
\hline Fase 4 & $\begin{array}{l}\text { Modelo de Banco de Dados - O Modelo de Banco de Dados descreve } \\
\text { todo o projeto de banco de dados do sistema. }\end{array}$ \\
\hline
\end{tabular}




\subsection{Planejamento}

Nesta fase uma nova metodologia baseada nos princípios do SCRUM foi construída para substituir o PDMS. Esta metodologia foi chamada de PDMS ági $]^{6}$ e foi construída com base em um aprofundado estudo sobre os princípios do SCRUM e alinhamento constante com a gestora da equipe de desenvolvimento de sistemas do TCE-MT. Foram feitos vários refinamentos entre a equipe de pesquisadores, que elaborou o PDMS ágil, a gestão e a equipe de TI até que o documento fosse considerado passível de implementação. Deste modo, obteve-se uma metodologia que apesar de ter base ágil, se adequou às necessidades da organização que estava habituada em seguir uma metodologia tradicional. As atividades que compõem o PDMS ágil são listadas na Tabela 2.

Tabela 2. Atividades do PDMS ágil

\begin{tabular}{|l|l|l|}
\hline Atividade & Ator/ Responsável & Definição \\
\hline Planejamento da Sprint & Product Own & $\begin{array}{l}\text { Planejar o Backlog da Sprint, } \\
\text { a equipe de desenvolvimento } \\
\text { escolhe suas atividades. }\end{array}$ \\
\hline Scrum Diária & Scrum Master & $\begin{array}{l}\text { Reunião diária para a equipe } \\
\text { saber o que cada integrante } \\
\text { realizou no dia anterior, o que } \\
\text { fará no presente dia e quais as } \\
\text { dificuldades que estão enfren- } \\
\text { tando. }\end{array}$ \\
\hline Revisão da Sprint & Scrum Master & $\begin{array}{l}\text { Revisão do que foi feito du- } \\
\text { rante a Sprint. }\end{array}$ \\
\hline Retrospectiva da Sprint & Scrum Master & $\begin{array}{l}\text { Scrum Master Processo de } \\
\text { avaliação do desenvolvi- } \\
\text { mento da Sprint. }\end{array}$ \\
\hline
\end{tabular}

Após vários refinamentos foi definido que os artefatos do PDMS ágil são:

1. Documento de Visão do Produto - O Documento de Visão do Produto estabelece as principais funcionalidades e fronteiras do sistema e registra informações que delimitam o projeto de desenvolvimento como um todo. Este artefato tem a função de um contrato entre os diferentes interessados no sistema (TCE, usuário gestor, usuário final etc.), registrando aquilo que será desenvolvido ao longo do projeto. Deve ser formalmente aprovado pelas partes envolvidas.

2. Backlog do Produto - O Backlog do Produto registra os principais requisitos a serem desenvolvidos até a entrega do produto. Este artefato lista os requisitos sem muitos detalhes e em ordem crescente dos mais importantes e a expectativa de conclusão.

3. Backlog da Sprint - O Backlog da Sprint registra o requisito a ser desenvolvido durante a sprint. Este artefato consta com uma breve história de usuário, critérios de aceitação, regras do negócio, protótipos e descrição das telas.

$6 \longdiv { \text { https: / / shortest.link/1obM } }$ 
4. Código-Fonte - O código-fonte representa qualquer trecho de código implementado ao longo do desenvolvimento do sistema.

Na Tabela 3 é mostrado um breve comparativo entre o PDMS anteriormente utilizado e o PDMS ágil:

Tabela 3. Comparativo entre as Metodologias

\begin{tabular}{|c|c|c|}
\hline & PDMS Tradicional & PDMS Ágil \\
\hline Metodologia & $\begin{array}{l}\text { RUP - metodologia para geren- } \\
\text { ciar projetos de desenvolvimento } \\
\text { de software orientados a objeto e } \\
\text { que usa a UML (Unified Modeling } \\
\text { Language- Linguagem de Modela- } \\
\text { gem Unificada) como uma das fer- } \\
\text { ramentas para especificação de sis- } \\
\text { temas }\end{array}$ & $\begin{array}{l}\text { SCRUM - metodologia para } \\
\text { gerenciar projetos de desen- } \\
\text { volvimento de software que } \\
\text { segue as práticas de: desen- } \\
\text { volvimento iterativo, geren- } \\
\text { ciamento de requisitos, de- } \\
\text { senvolvimento ágil, entrega } \\
\text { de software pronto em pouco } \\
\text { tempo. }\end{array}$ \\
\hline Implementação & $\begin{array}{l}\text { Representado através de modelos } \\
\text { de processos. Cada uma das } \\
4 \text { fases (Concepção, Elaboração, } \\
\text { Construção, Transição) }\end{array}$ & $\begin{array}{l}\text { Conta com o desenvolvi- } \\
\text { mento em Sprints, reuniões } \\
\text { de planejamento, retrospec- } \\
\text { tiva e revisão }\end{array}$ \\
\hline Fases & Fase 1 ; Fase 2 ; Fase 3 ; Fase 4. & $\begin{array}{l}1 \text { - Execução Sprints; } 2 \text { - Pla- } \\
\text { nejamento Sprint; } 3 \text { - Scrum } \\
\text { Diária; } 4 \text { - Revisão da Sprint; } \\
5 \text { - Retrospectiva da Sprint. }\end{array}$ \\
\hline Artefatos & $\begin{array}{l}1 \text { - Documento Visão do Produto; } 2 \\
\text { - Glossário; } 3 \text { - Matriz de requisi- } \\
\text { tos; } 4 \text { - Documento de arquitetura; } \\
5 \text { - Modelo de casos de uso; } 6 \text { - Mo- } \\
\text { delo de Banco de Dados; } 7 \text { - Código } \\
\text { Fonte; } 8 \text { - Compilação do Sistema } \\
\text { (build); } 9 \text { - Versão do sistema (re- } \\
\text { lease); 10- Pacote de Distribuição; } \\
\text { 11- Manuais do Sistema. }\end{array}$ & $\begin{array}{l}1 \text { - Documento Visão do Pro- } \\
\text { duto; 2- backlog do Produto; } \\
3 \text { - Backlog da Sprint; } 4 \text { - } \\
\text { Código fonte. }\end{array}$ \\
\hline
\end{tabular}




\begin{tabular}{|c|c|c|}
\hline Papeis & $\begin{array}{l}\text { 1 - Gestor do Projeto; } 2 \text { - Gerente } \\
\text { do Projeto; } 3 \text { - Analista de Siste- } \\
\text { mas; } 4 \text { - Analistas de Requisitos; } 5 \\
\text { - Analista Desenvolvedor; } 6 \text { - Pro- } \\
\text { jetista de Banco de Dados (DBA) } \\
7 \text { - Analista de Interface ; } 8 \text { - Líder } \\
\text { da Equipe ; } 9 \text { - Programador; } 10 \text { - } \\
\text { Programador (Desenvolvedor) ; } 11 \\
\text { - Analista Integrador ; } 12 \text { - Gerente } \\
\text { de Configuração ; } 13 \text { - Tester; } 14 \text { - } \\
\text { Líder do Projeto ; } 15 \text { - Demandante } \\
\text {; } 16 \text { - Analista da Qualidade ; } 17 \text { - } \\
\text { Usuário; } 18 \text { - Analista de Infraes- } \\
\text { trutura. }\end{array}$ & $\begin{array}{l}1 \text { - Dono do Produto; } 2 \text { - } \\
\text { Scrum Master }\end{array}$ \\
\hline
\end{tabular}

\subsection{Implementação da ação}

A implantação do PDMS ágil iniciou em novembro de 2020 com um mini-curso sobre os conceitos, as características e os papéis do SCRUM. Após isso, o Redmine foi ajustado para que os projetos executados com base no PDMS ágil fossem cadastrados. A partir daí todos os projetos demandados para a Secretaria de Sistemas de Informação do TCEMT passaram a ser desenvolvidos com base na nova metodologia. Assim os eventos, atividades e papéis do Scrum foram adotados e seguidos conforme definido no PDMS ágil. As sprints começaram para todas as coordenações ao mesmo tempo, de modo que todas começaram juntas e terminam juntas. No fim do dia é realizada uma retrospectiva geral do que foi realizado e do que precisa ser feito no outro dia. Com a execução das sprints e dos eventos os conceitos do SCRUM tem se consolidado na equipe.

\subsection{Avaliação}

Para se obter uma visão mais ampla da mudança do paradgma tradicional para o ágil no TCE-MT foi elaborado um questionário 7 no Google Forms. Nele os desenvolvedores expuseram suas opiniões a partir de perguntas relacionados à entrevista realizada com a gestora. Todas as perguntas foram de cunho descritivo, ficando a critério do entrevistado desenvolver sua resposta ou não. Abaixo constam as respostas dos desenvolvedores. No total 12 pessoas entre 20 desenvolvedores responderam o formulário. O nome dos mesmos não será divulgado para preservar o anonimato. Com relação a resistência à mudança de metodologia, 11 desenvolvedores responderam que não houve resistência e apenas 1 informou que houve, contudo essa pessoa não expressou o porquê da resistência. Com relação às desvantagens percebidas no uso do SCRUM, 6 pessoas não perceberam nenhuma, contudo as outras 6 mostraram suas opiniões relacionados à desvantagens, que foram:

- "A metodologia é muito proveitosa, porém da maneira que está sendo implementado no TCE, ainda está muito maçante. Precisa passar por melhorias e adaptação

https://forms.gle/wDePRKNsLweYGBNf8 
que custem menos tempo e seja mais prático. Por exemplo, algumas cerimônias estão sendo puladas."

- "As etapas da metodologia SCRUM não se adequaram 100\% a algumas equipes."

- "Talvez não se adeque a todas as áreas. Talvez para a nossa realidade tenhamos outras metodologias melhores."

Sobre o entendimento dos novos papéis da equipe, a resposta foi unânime. Todos enfatizaram que não houve dificuldade de entendimento, pois praticamente não houve alterações nas equipes. Já com relação aos benefícios percebidos no uso do SCRUM, 12 desenvolvedores responderam que houve:

- Alinhamento de comunicação da Equipe: Melhora de visão de backlog e demandas em andamento; Melhora na visão de atrasos e impeditivos.

- Maior Comunicação entre os colaboradores e Líder

- Melhor interação da equipe

- Melhor organização, maior transparência e maior conhecimento sobre o que é desempenhado de fato pela equipe.

- Melhor planejamento dos projetos, bem como melhor acompanhamento e também projetos concluídos mais rapidamente.

- Padronização dos métodos de trabalho.

Com relação aos indicadores para mensuração dos benefícios obtidos, se sabem o quanto ganharam em termos de tempo, de qualidade e de custo de desenvolvimento no uso do SCRUM, houve respostas divergentes. 8 desenvolvedores reportaram que ainda não há indicadores ou não sabem dizer se tem. Três desenvolvedores relataram que sim, há indicadores, mas não mencionaram quais são e nem qual a forma de mensuração, portanto, é provável que estes não tenham entendido a pergunta.

12 respondentes informaram que não sentiram falta de nenhuma documentação. Pelo contrário. 2 informaram que a forma de documentar as demandas ficou mais aprimorada e objetiva e houve mais facilidade do que dificuldade.

Em relação a adequação da metodologia ágil, se o PDMS foi aplicado a todos os projetos ou não, 2 pessoas não souberam responder. 7 desenvolvedores informaram que está sendo aplicado em todos os projetos. 3 alegaram que não está sendo implementado em todos os projetos.

Quando questionados sobre as possibilidades de melhorias na implementação do PSMD ágil, 3 pessoas informaram que não precisa de melhorias. Um deles acredita que o que foi implementado foi o melhor para a equipe. Os outros 9 informaram que há coisas a serem melhoradas, mas apenas 7 expuseram suas opiniões com mais detalhes, comos mostrado abaixo:

- “Algumas cerimônias não estão acontecendo - como a entrega das Sprints - "Sprint Review"";

- "Times pequenos com alto alinhamento de comunicação consideram a reunião desnecessária";

- "Alguns Produtos "Legados"que finalizam Sprints estão sem P.O. Isso dificulta boa parte do processo";

- "É necessário maior organização para realização de reuniões pois consomem muito tempo"; 
- "É necessário mais engajamento do pessoal";

- "Algumas equipes precisam de adequação no processo visto que a metodologia não se encaixa completamente nas tarefas dessas equipes";

- "Algumas equipes precisam de adequação no processo visto que a metodologia não se encaixa completamente nas tarefas dessas equipes";

- "Não foi realizada adequação do método de trabalho da equipe ao SCRUM";

- "Maior feedback da gestão, pois somente foi implantado e não tem acompanhamento se está melhorando/piorando etc".

Sobre a realização de treinamento em SCRUM para implantação do PDMS ágil, foi perguntado se o treinamento foi suficiente ou se sentem falta de um treinamento maior. 8 entrevistados responderam que o treinamento foi bom, suficiente ou satisfatório. 1 desenvolvedor relatou que não teve um treinamento oficial. Ele informou que um membro da equipe repassou as informações. Outros 3 desenvolvedores acharam que o treinamento foi pouco proveitoso, com muita teoria e pouca prática e um desses 3 relatou que sentiu falta de um treinamento maior.

Quando questionados sobre os principais desafios percebidos na utilização do SCRUM, os entrevistados responderam:

- O tempo curto da Sprint

- O tempo longo das reuniões que pela metodologia deveriam ser mais curtas.

- Adaptação ao processo como um todo.

- Maior documentação e registro de todas as atividades no Readmine.

- Aplicação da divisão dos papéis da metodologia SCRUM para uma equipe de trabalho reduzida.

\subsection{Documentação do aprendizado}

Foi identificada a necessidade de incluir user stories e de definir indicadores para mensurar os projetos baseados na metodologia ágil para se ter uma visão concreta dos benefícios. Também foi identificada a necessidade de se analisar quais projetos não se adequam a metodologia, conforme indicado por alguns desenvolvedores. Outras melhorias se referem a forma de conduzir os projetos de modo que de fato seja possível afirmar que o método ágil está sendo seguindo, por exemplo: gerir melhor o tempo das reuniões, realizar todas as cerimônias respeitando as características delas, engajar a equipe, solicitar maior transparência e feedback com relação ao acompanhamento dos projetos e da implantação do PDMS ágil. Percebe-se portanto, que estes não são ajustes a serem feitos na escrita da metodologia, mas são mudanças culturais a serem realizadas com treinamentos e com a aplicação diária dos corretos conceitos da metodologia ágil.

\section{Conclusão}

Este artigo reportou a elaboração e a implantação de uma metodologia ágil baseada em SCRUM, denominada de PDMS ágil, por meio de uma pesquisa-ação realizada no TCEMT. O artigo traz grandes inovações em termos de produto, pois o PDMS ágil substitui o PDMS tradicional baseado no RUP que era utilizado há anos pelo TCE-MT. Em termos de processo, a principal inovação é a nova forma de desenvolver software com base nos princípios de SCRUM escritos no PDMS ágil. Isto tem implicado, a partir das percepções dos desenvolvedores obtidas na fase de avaliação, ainda que não haja mensuração dos 
ganhos com a mudança de metodologia, em diminuição de tempo com projetos sendo concluídos mais rapidamente, em valorização de recursos humanos com a padronização dos trabalhos e melhor planejamento dos projetos e em aumento da qualidade dos sistemas desenvolvidos. A perspectiva dos desenvolvedores quanto à adoção e uso da metodologia serve de orientação para outras organizações que necessitem passar pela experiência de mudar do paradigma de desenvolvimento tradicional para o ágil. O PDMS ágil, artefato gerado pelos pesquisadores em conjunto com equipe de TI, consiste em uma importante contribuição para a literatura e pode ser útil como um ponto de partida para aqueles que quiserem construir sua própria metodologia ágil. Como trabalho futuro é planejado que o PDMS ágil seja ajustado conforme as lições aprendidas e documentadas. Métricas para acompanhamento de projetos baseados em SCRUM também estão sendo estudadas para serem adicionadas ao PDMS ágil. Os autores agradecem ao TCE-MT pela abertura e disponibilidade na realização da pesquisa e a unidade financiadora pelo financiamento do projeto.

\section{Referências}

Baskerville, R. L. (1999). Investigating information systems with action research. Communications of the association for information systems, 2(1):19.

Cooper, R. G. (2008). Perspective: The stage-gate ${ }^{\circledR}$ idea-to-launch process-update, what's new, and nexgen systems. Journal of product innovation management, 25(3):213-232.

de Souza Carvalho, W. C., Rosa, P. F., dos Santos Soares, M., da Cunha Jr, M. A. T., and Buiatte, L. C. (2011). A comparative analysis of the agile and traditional software development processes productivity. In 2011 30th International Conference of the Chilean Computer Science Society, pages 74-82. IEEE.

Hidalgo, E. S. (2019). Adapting the scrum framework for agile project management in science: case study of a distributed research initiative. Heliyon, 5(3):e01447.

Lappi, T. and Aaltonen, K. (2017). Project governance in public sector agile software projects. International Journal of Managing Projects in Business.

Merriam, S. B. and Tisdell, E. (2009). Qualitative research: A guide to design and implementation. san francisco, ca: Lohn wiley \& sons.

Nicholls, G. M., Lewis, N. A., and Eschenbach, T. (2015). Determining when simplified agile project management is right for small teams. Engineering Management Journal, 27(1):3-10.

Noordeloos, R., Manteli, C., and Van Vliet, H. (2012). From rup to scrum in global software development: A case study. In 2012 IEEE Seventh International Conference on Global Software Engineering, pages 31-40. IEEE.

Oliveira, R. L. F. and Pedron, C. D. (2021). Métodos ágeis: Uma revisão sistemática sobre benefícios e limitações. Brazilian Journal of Development, 7(1):4520-4534.

Schwaber, K. (2004). Agile project management with Scrum. Microsoft press.

Shafiee, S., Wautelet, Y., Hvam, L., Sandrin, E., and Forza, C. (2020). Scrum versus rational unified process in facing the main challenges of product configuration systems development. Journal of Systems and Software, 170:110732. 